[9] K. Györy, Explicit upper bounds for solutions of some diophantine equations, ibid. 5 (1980), pp. $3-12$.

[10] - Résultats effectifs sur la représentation des entiers par des formes décomposables, Queen's Papers in Pure and Applied Math., No, 56. Kingston, Canada, 1980.

[11] - On the representation of integers by decomposable forms in several variables, Publ. Math. Debrecen 28 (1981), pp. 89-98.

[12] - On S-integral solutions of norm form, discriminant form and index form equations, Studia Sci. Math. Hungar. 16 (1981), pp. 149-161.

[13] - Bounds for the solutions of norm form, discriminant form and index form equations in finitely generated integral domains, Acta. Math. Hungar. 42 (1983), pp. 45-80.

[14] - On norm form, discriminant form and index form equations, in: Topics in Classical Number Theory, Coll. Math. Soc. J. Bolyai 34, North Holland Publ. Comp., Amsterdam etc., 1984 , pp. 617-676.

[15] K. Györy and Z.Z. Papp, Effective estimates for the integer solutions of norm form and discriminant form equations, Publ. Math. Debrecen 25 (1978), pp. 311-325.

[16] S. V. Koto v, The Thue-Mahler equation in relative fields (Russian), Acta. Arith. 27 (1975), pp. $293-315$.

[17] S. Lang, Fundamentals of diophantine geometry, Springer Verlag, New York etc., 1983.

[18] J. B. Rosser and L. Schoenfeld, Approximate formulas for some functions of prime numbers, Illinois J. Math. 6 (1962), pp. 64-94.

[19] T. N. Shorey, A. J. van der Poorten, R. Tijdeman and A. Schinzel, Applications of the Gelfond-Baker method to diophantine equations, in: Transcendence Theory: Advances and Applications, Academic Press, London etc., 1977, pp. 59-77.

[20] T. N. Shorey and R. Tijdeman, Exponential diophantine equations, Cambridge University Press, 1987.

[21] V. G. Sprindžuk, Classical diophantine equations in two unknowns (Russian), Nauka Moskva, 1982.

[22] B. L. van der Waerden, Algebra 1, 7. Auff., Springer Verlag, Berlin etc., 1966.

\title{
DEPARTMENT OF PURE MATHEMATICS
}

CENTRE FOR MATHEMATICS

1098 SI Amsterdam

The Netherlands

MATHEMATICAL INSTITUTE

MOSSUTH LATOS UNTYERE

KOSSUTH LAJOS UNTVERSITY

4010 Debrecen

SCHOOL OF MATHEMATICS

TATA INSTITUTE OF FUNDAMENTAL RESEARCH

Bombay 400005

India

MATHEMATICAL INSTITUTE

UNIVERSTTY LEIDEN

2300 RA Leiden

\section{On $S$-integral solutions of the Catalan equation}

by

\section{B. BRINDZA (Debrecen)}

1. Introduction. In $1976 \mathrm{R}$. Tijdeman [15], employing a refined form of an inequality of A. Baker [1] on linear forms in logarithms, gave an effectively computable bound for the solutions of the Catalan equation. Later, A. J. van der Poorten proved the following $p$-adic generalization of Tijdeman's result.

Theorem A (A. J. van der Poorten [12]). Let $S$ be a finite set of distinct positive primes, $S=\left\{p_{1}, \ldots, p_{s}\right\}$. Then there is an effectively computable constant $C_{1}$ depending only on the set $S$, such that all rational integer solutions $x>1, y>1, u>1, v>1, \omega_{1}, \ldots, \omega_{s}$ with $(x, y)=1$ and $u v>4$ of the equation

$$
x^{\mu}-y^{\dot{v}}=\left(p_{1}^{\omega_{1}} \ldots p_{s}^{\omega_{S}}\right)^{\left\{\mu_{s} v\right\}}
$$

are bounded by $C_{1}$.

(We denote by $(x, y)$ the g.c.d. of integers $x, y$ and by $\{u, v\}$ the 1.c.m. of integers $u, v$.)

Let $K$ be an algebraic number field with ring of integers $\mathcal{O}_{\boldsymbol{K}}$. Further, let $\mid \alpha$ denote the maximum absolute value of the conjugates of an algebraic number $\alpha$. Recently, K. Györy, R. Tijdeman and the author have extended Tijdeman's result to the case of algebraic number fields.

Theorem B (B. Brindza, K. Györy, R. Tijdeman [3]). There exists an effectively computable number $C_{2}$ which depends only on $\boldsymbol{K}$ such that all solutions of the equation

$$
x^{p}-y^{q}=1 \quad \text { in } \quad x, y \in \mathcal{O}_{\mathbf{K}} ; p, q \in N
$$

with $x, y$ not roots of unity and $p>1, q>1, p q>4$ satisfy

$$
\max \left\{[x, \mid y, p, q\}<C_{2}\right. \text {. }
$$

For further results connected with the Catalan equation we refer to Shorey and Tijdeman [14], Ribenboim [13] and Tijdeman [15]; [16].

Let $\mathfrak{p}_{1}, \ldots, \mathfrak{p}_{t}(t \geqslant 0)$ be distinct prime ideals in $K$, let $P=\max N \mathfrak{p}_{i}$ (with 
$P=1$ if $t=0$ ) and let $S$ denote the set of all additive valuations of $K$ corresponding to $p_{1}, \ldots, p_{t}$. Further, let $c_{K, S}$ denote the ring of $S$-integers of $\boldsymbol{K}$. We recall that an element $\alpha$ of $\boldsymbol{K}$ is said to be $S$-integral if $v(\alpha) \geqslant 0$ for all valuations $v$ of $K$ not contained in $S$. The purpose of this paper is to prove the following result.

THEOREM. There exists an effectively computable constant $C$ which depends only on $K, P$ and $t$ such that all solutions of the equation (1) in $x, y \in \mathcal{O}_{K, S}, p, q \in N$ with $p, q>1, p q>4$ and $x, y$ not roots of unity satisfy

$$
\max \{H(x), H(y), p, q\}<C .\left(^{1}\right)
$$

We note that the proof of this theorem is also based on the Gel'fondBaker method and we shall use some arguments from Tijdeman's proof [15], and the proofs of Theorem A and Theorem B.

I would like to thank Professor K. Györy and Professor R. Tijdeman for their suggestions and valuable remarks.

2. Auxiliary results. Let $\alpha_{1}, \ldots, \alpha_{k}(k>1)$ be algebraic numbers in $K$ with heights at most $A_{1}, \ldots, A_{k}$ respectively, and assume that $A_{j} \geqslant 4$, $1 \leqslant j \leqslant k$. Put

$$
\Omega^{\prime}=\prod_{i=1}^{k-1} \log A_{i}, \quad \Omega=\Omega^{\prime} \log A_{k}, \quad n=[K: Q] .
$$

LEMMA 1 (A. Baker [1]). There exist effectively computable constants $C_{3}>0$ and $C_{4}>0$ such that the inequalities

$$
0<\left|\alpha_{1}^{b_{1}} \ldots \alpha_{k}^{b_{k}}-1\right|<\exp \left\{-\left(C_{3} k n\right)^{C_{4} k} \Omega \log \Omega^{\prime} \log B\right\}
$$

have no solutions in rational $b_{1}, \ldots, b_{k}$ with absolute values at most $B(\geqslant 2)$.

Denote by $\mathfrak{p}$ a prime ideal of $\boldsymbol{K}$ and suppose that $\mathfrak{p}$ divides the rational prime $p$.

Lemma 2 (A. J. van der Poorten [12]). For some effectively computable number $C_{p}^{*}>0$ depending only on $p, k$ and $n$ the inequalities

$$
0<\left|\alpha_{1}^{b_{1}} \ldots \alpha_{k}^{b_{k}}-1\right|_{p}<\exp \left\{-C_{p}^{*} \Omega \log \Omega^{\prime}(\log B)^{2}\right\}
$$

have no solutions in rational integers $b_{1}, \ldots, b_{k}$ with absolute values at most $B$ $(\geqslant 4)$. (See "Added in proff", page 411.)

(Let $v_{p}$ denote the additive valuation of $\boldsymbol{K}$ corresponding to $\mathfrak{p}$ and $e_{\mathrm{p}}$ is the exponent to which $p$ divides $p$, moreover, $f_{\mathfrak{p}}$ is given by $N p=p^{S p}$. If $\alpha$ is any non-zero element of $\boldsymbol{K}$ then $|\alpha|_{p}=(N \mathfrak{p})^{-v_{p}(\alpha) / e_{p} f_{p}}$.

Let $\beta_{1}, \ldots, \beta_{n}, \pi_{1}, \ldots, \pi_{s}(n \geqslant 2, s \geqslant 0)$ be algebraic integers in $\boldsymbol{K}$ with $\beta_{i} \neq \beta_{j}$ for $i \neq j$ and suppose that $0 \neq \pi_{i}$ is not a unit in $K, 1 \leqslant i \leqslant s$.

(1) By the height $H(\alpha)$ of an algebraic number $\alpha$ we mean the maximum of the absolute values of the relatively prime integer coefficients in its minimal defining polynomial over $Z$.

Put

$$
\begin{gathered}
S_{1}=\left\{\pi_{1}^{k_{1}} \ldots \pi_{s}^{k_{s}} \mid 0 \leqslant k_{i} \in Z, i=1, \ldots, s\right\} \\
f(X, Y)=\prod_{i=1}^{n}\left(X-\beta_{i} Y\right)^{r_{i}} .
\end{gathered}
$$

Consider the equation

$$
f(x, z)=\varepsilon \gamma y^{m}
$$

where $z, \gamma \in S, x \in \mathcal{O}_{K}, m \in N, \varepsilon$ is a unit and $0 \neq y \in \mathcal{O}_{K}$ is not a unit. Let $\tau$ be a positive number.

LEMMA 3 (Shorey and Tijdeman [14], Th. 10.3). If

$$
\min \left\{\operatorname{ord}_{\mathrm{p}} x, \operatorname{ord}_{\mathrm{p}} z\right\} \leqslant \tau
$$

for all prime ideals $\mathfrak{p}$ then all solutions of the equation $f(x, z)=\varepsilon \gamma y^{m}$ in $x, z, \varepsilon$, $\gamma, y, m$ with above mentioned conditions satisfy $m<C_{5}$, where $C_{5}$ is an effectively computable constant depending on $K, S_{1}, \tau$ and the binary form $f$.

The follwing lemma is an effective version of a well-known theorem of LeVeque [11].

Lemma 4 (B. Brindza [2]). Let

$$
f(x)=a \prod_{i=1}^{n}\left(X-\alpha_{i}\right)^{r_{i}} \in K[X]
$$

be a polynomial with $a \neq 0$ and $\alpha_{i} \neq \alpha_{j}$ for $i \neq j$. Further, let $m$ be $a$ positive integer and put $t_{i}=m /\left(m, r_{i}\right)$ for $i=1, \ldots, n$. Suppose that $t_{1}, \ldots, t_{n}$ is not a permutation of the n-tuples $\{t, 1, \ldots, 1\}$ and $\{2,2,1, \ldots, 1\}$. Then all solutions $x, y \in \mathcal{O}_{\mathbf{K}, s}$ of the equation

satisfy

$$
f(x)=y^{m}
$$

$$
\max \{H(x), H(y)\}<\exp \exp \left\{C_{6} P^{2}(s+1)^{3}\right\}
$$

where $C_{6}$ is an effectively computable constant depending only on $K, f$ and $\boldsymbol{m}$.

LEMMA 5. There are independent units $\varepsilon_{1}, \ldots, \varepsilon_{r}$ in $\boldsymbol{K}$ ( $\boldsymbol{r}$ denotes the unit rank of $\boldsymbol{K}$ ) and a root of unity $\varepsilon_{0}$ such that

$$
\max _{i} \mid \varepsilon_{i}<C_{7}, \quad \varepsilon_{0}<C_{8}
$$

and that every unit can be written as $\varepsilon=\varepsilon_{0}^{a_{0}} \varepsilon_{1}^{a_{1}} \ldots \varepsilon_{r}^{a_{r}}$ with $a_{0}, \ldots, a_{r} \in \boldsymbol{Z}$ where $C_{7}$ and $C_{8}$ are effectively computable numbers depending only on $K$.

For a proof see [15, Corollaries A.4 and A.5] or [8, Lemma 3].

There are $n=[K: Q]$ isomorphisms $\sigma_{1}, \ldots, \sigma_{n}$ of $K$ into the complex numbers; denote the images of an element $\alpha$ of $\boldsymbol{K}$ under these isomorphisms by

$$
\sigma_{i}(\alpha)=\alpha^{(i)} \quad \text { for } \quad i=1, \ldots, n
$$


Lemma 6. Let $0 \neq \alpha \in \mathbb{K}$ with $\left|N_{\mathbf{K} / \boldsymbol{Q}}(\alpha)\right|=M$. Then there exists a $\beta \in \boldsymbol{K}$ associated to $\alpha$ such that

$$
\left|\log \left(M^{-1 / n}\left|\beta^{(i)}\right|\right)\right| \leqslant C_{9} \quad \text { for } \quad i=1, \ldots, n
$$

where $C_{9}$ is an effectively computable number which depends only on $\boldsymbol{K}$.

For a proof see [15, Lemma A.15] or [8, Lemma 3].

LEMMA 7. There is an integral basis $\omega_{1}, \ldots, \omega_{n}$ of $\boldsymbol{K}$ such that

$$
\max _{i}\left|\omega_{i}\right|<C_{10} \mid D_{k} C^{C_{11}}
$$

where $D_{\mathrm{K}}$ is the discriminant of $K$ and $C_{10}, C_{11}$ are effectively computable constants depending only on $n$.

(Cf. K. M. Bartz, On a theorem of Sokolovski, Acta Arith. 34 (1978), pp. 113-126.)

The following lemma is a special case of a result of Gyôry (see Lemma 6 in [10]).

LeMmA 8. Let $U_{s}$ denote the group of $S$-units in $\boldsymbol{K}$. If $x_{1}, x_{2}$ and $x_{3}$ are non-zero algebraic integers in $\boldsymbol{K}$ satisfying

$$
x_{1}+x_{2}+x_{3}=0 \text { and } x_{1}, x_{2}, x_{3} \in U_{s} \cap \mathcal{O}_{K}
$$

then for some $o \in U_{s} \cap \mathscr{O}_{\mathbf{K}}$ and $\varrho_{j} \in \mathcal{O}_{\mathbf{K}}$ we have $x_{j}=o \varrho_{j},(j=1,2,3)$ and $\max \left|\varrho_{j}\right|<C_{12}$ where $C_{12}$ is an effectively computable constant depending only on $\boldsymbol{K}$ and $S$.

LEMMA 9. Let $\alpha$ be a non-zero algebraic integer of degree $n$ which is not a root of unity. There exists an effectively computable positive number $C_{13}$ depending only on $n$ such that

$$
|\alpha|>1+C_{13} \text {. }
$$

Proof. This theorem is due to Schinzel and Zassenhaus who gave an explicit value for $C_{13}$. See also Cantor and Straus [4] and Dobrowolski [7].

3. Proof of the theorem. Let $v$ be an arbitrary (additive) valuation of $\boldsymbol{K}$. It is well known that if $v(\alpha) \neq v(\beta)$ for some $\alpha, \beta \in \boldsymbol{K}$ then

$$
v(\alpha+\beta)=\min \{v(\alpha), v(\beta)\} .
$$

Suppose that $v\left(x^{p}\right)<0$ or $v\left(y^{q}\right)<0$. Then from equation (1) we have $v\left(x^{p}\right)$ $=v\left(y^{q}\right)$. It means that the principal ideals $\left[x^{p}\right]$ and $\left[y^{q}\right]$ can be written in the following form

$$
[x]^{p}=\mathfrak{X} /\left(\mathfrak{p}_{1}^{\alpha_{1}} \ldots \mathfrak{p}_{s}^{\alpha_{s}}\right)^{\{p, q\}}, \quad[y]^{q}=\mathfrak{Y} /\left(\mathfrak{p}_{1}^{\alpha_{1}} \ldots \mathfrak{p}_{s}^{\alpha_{s}}\right)^{\{p, q\}}
$$

where $\mathfrak{X}$ and $\mathfrak{Y}$ are integral ideals, $\alpha_{1}, \ldots, \alpha_{s}$ are positive integers such that $\mathfrak{X} \mathfrak{Y}, \mathfrak{p}_{1}^{\alpha_{1}} \ldots \mathfrak{p}_{s}^{\alpha_{s}}$ are relatively prime. We may assume that $0<s(\leqslant t)$. Supposing the contrary we get $x, y \in \mathcal{O}_{K}$ and we can apply Theorem $B$.
It is known that there are infinitely many prime ideals in every ideal class of $K$. Hence we can choose distinct prime ideals $q_{1}^{\prime}, \ldots, q_{s}^{\prime}$ such that $\mathfrak{p}_{i} \mathfrak{q}_{i}^{\prime}=\left[f_{i}\right](i=1, \ldots, s)$ for some $f_{i} \in \mathcal{O}_{\mathbf{K}}$ and $\mathfrak{p}_{i} \|\left[f_{i}\right]$ but $p_{j} \backslash\left[f_{i}\right]$ for $j \neq i$. Let $\omega_{1}, \ldots, \omega_{n}$ be an integral basis of $\boldsymbol{K}$ such that

$$
\max _{i}\left\lceil\left.\omega_{i}\left|<C_{10}\right| D_{k}\right|^{c_{11}}=: c_{1}\right. \text {. }
$$

In the proof $c_{1}, c_{2}, \ldots$ denote effectively computable positive constants which depend only on $K, P$ and $t$. Write $N p_{i}=n_{i}(1 \leqslant i \leqslant s)$ and

$$
f_{i}=x_{1 i} \omega_{1}+\ldots+x_{n i} \omega_{n} \quad(1 \leqslant i \leqslant s)
$$

where $x_{j i} \in Z$ for $1 \leqslant j \leqslant n, 1 \leqslant i \leqslant s$. Let $x_{j i}^{\prime}$ be defined by

$$
x_{j i}^{\prime} \equiv x_{j i}\left(\bmod n_{1}^{2} \ldots n_{s}^{2}\right), \quad 0 \leqslant x_{j i}^{\prime}<n_{1}^{2} \ldots n_{s}^{2}
$$

for every pair $(j, i)$ and

$$
f_{i}^{\prime}=x_{1 i}^{\prime} \omega_{1}+\ldots+x_{n i}^{\prime} \omega_{n}, \quad i=1, \ldots, s .
$$

Then $\prod_{i=1}^{s} p_{l}^{2} \mid\left[f_{i}-f_{i}^{\prime}\right]$ and $p_{i} \|\left[f_{i}^{\prime}\right]$ but $\mathfrak{p}_{j} \nmid\left[f_{i}^{\prime}\right]$ for $j \neq i$. We can write $\left[f_{i}^{\prime}\right]=\mathfrak{p}_{i} \mathfrak{q}_{i}$ tor some integral ideal $\mathfrak{q}_{i}$ such that $\mathfrak{p}_{1}, \ldots, \mathfrak{p}_{s}$ and $\mathfrak{q}_{1}, \ldots, \mathfrak{q}_{s}$ are relatively prime ideals and

$$
N \mathrm{q}_{i}<\left|N\left(f_{i}^{\prime}\right)\right| \leqslant\left[H\left(f_{i}^{\prime}\right)\right]^{n} \leqslant\left(2\left|f_{i}^{\prime}\right|\right)^{n^{2}}=: c_{2}
$$

Let $h=h_{\mathbf{X}}$ denote the class number of $\boldsymbol{K}$ and $0 \leqslant m_{i}<h$ such that $m_{i} \equiv \alpha_{i}(\bmod h), i=1, \ldots, s$. Then

$$
\mathfrak{p}_{1}^{\alpha_{1}} \ldots \mathfrak{p}_{s}^{\alpha_{s}} q_{1}^{m_{1}} \ldots \mathfrak{q}_{s}^{m_{s}}=[z]
$$

for some $z \in \mathcal{O}_{\mathbf{K}}$ and by Lemma 6 we may assume that $|z|<c_{3}|N(z)|$. Putting

$$
x_{1}=x \cdot z^{\{p, q\} p^{-1}} \quad \text { and } \quad y_{1}=y \cdot z^{\{p, q\} q^{-1}}
$$

we have

and

$$
x_{1}^{p}-y_{1}^{q}=z^{\{p, q\}}, \quad x_{1}, y_{1} \in \mathscr{O}_{\mathbf{K}}
$$

$$
\left(\left[x_{1}^{p}\right],[z]^{\{p, q\}}\right)=\left(\left[y_{1}^{q}\right],[z]^{\{p, q\}}\right)=\left(q_{1}^{m_{1}} \ldots \mathfrak{q}_{s}^{m_{s}}\right)^{\{p, q\}} .
$$

Put $\mathfrak{p}_{i}^{h}=\left[\pi_{i}\right], i=1, \ldots, s$ and $\left(q_{1}^{m_{1}} \ldots \mathfrak{q}_{s}^{m_{s}}\right)^{h}=[\vartheta]$ with some fixed $\pi_{1}, \ldots, \pi_{s}$, $\vartheta \quad$ and $q \alpha_{i}=h \alpha_{i}^{(1)}+\alpha_{i}^{(2)}, \quad q=a h+b \quad$ with $0 \leqslant \alpha_{i}^{(2)}<h, \quad 0 \leqslant b<h$ $\left(\alpha_{i}^{(1)}, \alpha_{i}^{(2)}, a, b \in Z\right)$. By Lemma 6 we can write

$$
z^{q} \vartheta^{-a}=\pi_{1}^{\alpha_{1}} \ldots \pi_{s}^{\alpha_{s}}
$$

where $\varepsilon$ is a unit and

$$
|\varphi|<c_{4}|N(\varphi)|<c_{4} \prod_{i=1}^{s}\left(N p_{i} \mathfrak{q}_{i}^{m_{i}}\right)^{h}
$$


At first we assume that $p=q(>2)$. From (2) we obtain

$$
x_{1} y_{1}^{q}=y_{1}^{q}\left(y_{1}^{q}+z^{q}\right) \text {. }
$$

For brevity let us set $y_{2}=y_{1}^{a} \vartheta^{-a}, y_{3}=x_{1}^{h} y_{1}^{h} \vartheta^{-2}$ and $\gamma=\vartheta^{2 b}$. 7 $y_{2}, y_{3} \in \mathcal{O}_{\mathbf{K}}$ and

$$
\gamma y_{3}^{\natural}=y_{2}^{h}\left(y_{2}+z^{q} \vartheta^{-a}\right)^{h} .
$$

Hence, by (3) we have

$$
\gamma \varepsilon^{-2 h} y_{3}^{q}=\left(y_{2} \varepsilon^{-1}\right)^{h}\left(y_{2} \varepsilon^{-1}+\pi_{1}^{\alpha_{1}^{(1)}} \ldots \pi_{s}^{\alpha_{s}^{(1)}} \varphi\right)^{h}
$$

Moreover, $y_{2} \varepsilon^{-1}$ and $\pi_{1}^{\alpha_{1}^{(1)}} \ldots \pi_{s}^{\alpha_{s}^{(1)}}$ are relatively prime integers in $\mathcal{O}_{\mathbf{K}}$. I is not a unit then from Lemma 3 we get $q<c_{5}$. We suppose now $y_{3}=\left(x_{1}^{h} \vartheta^{-1}\right)\left(y_{1}^{h} \vartheta^{-1}\right)$ is a unit. It means that $\varepsilon_{1}=x_{1}^{h} \vartheta^{-1} \in \mathcal{O}_{\mathbf{K}}$ anc $=y_{1}^{h} \vartheta^{-1} \in \mathcal{O}_{\mathbf{K}}$ are also units. Therefore $\mathfrak{q}_{1}^{m_{1}} \ldots \mathfrak{q}_{s}^{m_{s}}$ and $\mathfrak{p}_{1}^{\alpha_{1}} \ldots p_{s}^{\alpha_{s}}$ are princ

$$
\mathfrak{q}_{1}^{m_{1}} \ldots \mathfrak{q}_{s}^{m_{s}}=[Q], \quad \mathfrak{p}_{1}^{\alpha_{1}} \ldots \mathfrak{p}_{s}^{\alpha_{s}}=[R]
$$

with some $Q, R \in \mathcal{O}_{\mathbb{K}}$. From (2) we obtain

$$
\varepsilon_{3}^{4}-\varepsilon_{4}^{q}=\varepsilon R^{q}
$$

where $\varepsilon_{3}=x_{1} Q^{-1}, \varepsilon_{4}=y_{1} Q^{-1}$ and $\varepsilon$ are units. Thus, by Lemma 8 we

We infer

$$
\mid \overline{R^{q} \varepsilon \varepsilon_{4}^{-q}}<c_{6}
$$

hence $q<c_{7}$.

$$
2^{q} \leqslant\left|N\left(R^{q} \varepsilon \varepsilon_{4}^{-q}\right)\right|<\left(2 c_{6}\right)^{n^{2}}
$$

It suffices to prove the theorem in case $p$ and $q$ are primes such $p q>4$. Indeed, let $p_{1}$ and $q_{1}$ be the greatest prime factor of $p$ and $q$. The implies

$$
\left(x^{p / p_{1}}\right)^{p_{1}}-\left(y^{q / q_{1}}\right)^{q_{1}}=1 .
$$

Hence, if $p_{1} q_{1}>4$ then $\max \left\{p_{1}, q_{1}\right\}<C$ and

$$
\max \left\{H\left(x^{p / p_{1}}\right), H\left(y^{q / q_{1}}\right)\right\}<C .
$$

There are $A, B \in Z$ such that $\max \{|A|,|B|\}<C$ and $A x^{p / p_{1}}, B y^{q / a_{1}}$ integers in $K$. But then $p_{1}^{p / p_{1}} \mid[A]$ and $\mathfrak{p}_{1}^{q / q_{1}} \mid[B]$. It means $\max \left\{p / p_{1}, q / q_{1}\right\}<C^{\prime}$. In the remaining case we have $p_{1}=q_{1}=2$ anc can write

$$
\left(x^{2^{\alpha}}\right)^{4}-\left(y^{2^{\beta}}\right)^{2}=1 \quad \text { or } \quad\left(x^{2^{\beta}}\right)^{2}-\left(y^{2^{\alpha}}\right)^{4}=1
$$

for some non-negative integers $\alpha, \beta$ and by Lemma 4

$$
\max \left\{H\left(x^{2^{\alpha}}\right), H\left(y^{2^{\beta}}\right)\right\}<c_{8} \quad \text { or } \max \left\{H\left(x^{2^{\beta}}\right), H\left(y^{2^{\alpha}}\right)\right\}<c_{8} .
$$

Consequently, as above, $\max \{H(x), H(y), \alpha, \beta\}<c_{9}$. After this, we may assume without loss of generality that $p$ and $q$ are distinct primes and $p q>4$. get

Now suppose that $p$ is fixed. Put $L=K(\eta)$ with $\eta=e^{2 \pi i / p}$. From (2) we

$$
y_{1}^{q}=\prod_{i=1}^{p}\left(x_{1}-\eta^{i} z^{q}\right) .
$$

Using the notation of the case $p=q$ we obtain

$$
\vartheta^{b p} y_{1}^{q h}=\vartheta^{p q} \prod_{i=1}^{p}\left(x_{1} \vartheta^{-a}-\eta^{i} z^{q} \vartheta^{-a}\right)^{h} .
$$

Putting $\gamma_{1}=\vartheta^{b p}, y_{2}=y_{1}^{h} \vartheta^{-p}$ and $x_{2}=x_{1} \vartheta^{-a}$ we have $y_{2}, x_{2} \in \mathcal{O}_{\mathbf{K}}$ and

$$
\gamma_{1} y_{2}^{q}=\prod_{i=1}^{p}\left(x_{2}-\eta^{i} z^{q} \vartheta^{-a}\right)^{h}
$$

hence

$$
\gamma_{1} \varepsilon^{-p h} y_{2}^{q}=\prod_{i=1}^{p}\left(x_{2} \varepsilon^{-1}-\eta^{i} \pi_{1}^{\alpha_{1}^{(1)}} \ldots \pi_{s}^{\alpha_{s}^{(1)}} \varphi\right)^{h}
$$

where $\varepsilon$ is a unit in $K$ and $|\varphi|<c_{10}$ again. Further, $x_{2} \varepsilon^{-1}$ and $\pi_{1}^{\alpha_{1}^{(1)}} \ldots \pi_{s}^{\alpha_{s}^{(1)}}$ are relatively prime integers in $\mathscr{O}_{K}$ (and in $\mathcal{O}_{L}$ of course). If $y_{2}$ is a unit in $\mathscr{O}_{L}$, respectively $\mathcal{O}_{\mathrm{K}}$ then $y_{1}^{h}$ and $\vartheta^{p}$ are associated and $\left[y_{1}\right]=q_{1}^{m_{1} p} \ldots \mathfrak{q}_{s}^{m_{s} p}$. Since $y_{1}^{q}$ and $z^{p q}$ have only fixed prime ideals, by Lemma 5 and Lemma 6 we can write

$$
y_{1}^{q}=f_{1} y_{3}^{3 p} \quad \text { and } \quad z^{p q}=f_{2} z_{1}^{3 p}
$$

where $f_{1}, f_{2}, y_{3}$ and $z_{1}$ are integers in $K$ such that

$$
\max _{i}\left\{\left[f_{i}\right\}<c_{11}\right. \text {. }
$$

Then $\xi_{1}=y_{3}^{p} z_{1}^{-p}$ and $\xi_{2}=x_{1} z_{1}^{-3}$ are non-zero $S^{\prime}$ integers, where $S^{\prime}$ is the set of all valuations of $\boldsymbol{K}$ corresponding to the prime ideal divisors of the product $q_{1} p_{1} \ldots q_{s} p_{s}$. From (2) we obtain

$$
\xi_{2}^{p}=f_{1} \xi_{1}^{3}+f_{2}
$$

We may apply Lemma 4 to (4) and we get $H\left(\xi_{1}\right)<c_{12}$. We deduce from definition of $\xi_{1}$ that

$$
H\left(y_{1}^{q} / z^{p q}\right)<c_{13} .
$$


Then there is an $a \in Z$ such that $|a|<c_{14}$ and $a y_{1}^{q} / z^{p q}$ is an integer in $K$. Since $\mathfrak{p}_{1} \mid[z]$ and $\mathfrak{p}_{1} \chi\left[y_{1}\right]$, we have $p_{1}^{p q} \mid[a]$ and hence $q<c_{15}$. If $y_{2}$ is not a unit in $\mathcal{O}_{\mathbf{L}}$ then, by Lemma $3, q<c_{16}$.

It is easy to verify that if $q$ is fixed then we can apply similar arguments. The equation (1) can be written

$$
(-y)^{q}-(-x)^{p}=1 \quad(p, q>2)
$$

Thus we may assume that $p$ and $q$ are primes such that $p>q>c_{17}$, where $c_{17}$ is large enough.

We have by (2)

$$
\left[y_{1}\right]^{q}=\left[x_{1}-z^{q}\right]\left[\alpha\left(x_{1}-z^{q}\right)+p z^{p q}\right]
$$

for some $\alpha \in \mathcal{O}_{\mathbf{K}}$. We can write

$$
[p]=P_{1}^{a_{1}} \ldots P_{w}^{a_{w}} \quad \text { and } \quad[q]=Q_{1}^{b_{1}} \ldots Q_{t}^{b_{t}}
$$

where $P_{1}, \ldots, P_{w}, Q_{1}, \ldots, Q_{t}$ are distinct prime ideals in $K ; w, t, a_{1}, \ldots, a_{w}$, $b_{1}, \ldots, b_{t}$ are positive integers not exceeding $n$. If $c_{17}$ is large enough then $(p q, z)=1$ and if $p^{k}$ is a common divisor of $\left[x_{1}-z^{q}\right]$ and $\left[\alpha\left(x_{1}-z^{q}\right)+p z^{p q}\right]$ for some prime ideal $\mathfrak{p}$ and positive integer $k$, but $\mathfrak{p}_{1} \chi[z]$ then $\mathfrak{p}^{k} \mid[p]$ and $k \leqslant n$. Hence the ideal $\left[x_{1}-z^{q}\right]$ can be written in the following form

$$
\left[x_{1}-z^{q}\right]=\mathfrak{q}_{1}^{n_{1}} \ldots \mathfrak{q}_{s}^{n_{s}} P_{1}^{k_{1}} \ldots P_{w}^{k_{w}} \mathfrak{X}_{1}^{q}
$$

where $\mathfrak{X}_{1}$ is an integral ideal $n_{1}, \ldots, n_{s}, k_{1}, \ldots, k_{w}$ are rational integers such that $\left|k_{i}\right| \leqslant n$ and $0 \leqslant n_{j}$. Further, we may assume that $n_{j}<q(j=1, \ldots, s)$. (The factor $q_{i}^{\left[n_{i} / q\right]}$ can be multiplied to $\mathfrak{X}_{1}$.) Setting $q_{i}^{h}=\left[\vartheta_{i}\right], \mathfrak{X}_{1}^{h}=\left[X_{1}\right]$, $P_{i}^{h}=\left[u_{i}\right]$ for some $X_{1}, u_{1}, \ldots, u_{w}, \vartheta_{1}, \ldots, \vartheta_{s} \in \mathcal{O}_{\mathbf{K}}$ we obtain

$$
\left(x_{1}-z^{q}\right)^{h}=\varepsilon \vartheta_{1}^{n_{1}} \ldots \vartheta_{s}^{n_{s}} u_{1}^{k_{1}} \ldots u_{w}^{k_{w}} X_{1}^{q}
$$

where $\varepsilon$ is a unit in $K$. By using Lemma 6 we can choose $y_{1}, \ldots, \vartheta_{s}$, $u_{1}, \ldots, u_{w}$ such that

$$
\max \left\{[ \vartheta _ { 1 } , \ldots , \sqrt { \vartheta _ { s } } \} < c _ { 1 8 } \quad \text { and } \operatorname { m a x } \left\{\left[u_{1}, \ldots, \mid u_{w}\right\}<c_{19} p\right.\right. \text {. }
$$

By applying Lemma 5 we can write

$$
\left(x_{1}-z^{q}\right)^{h}=\varepsilon_{0}^{m_{0}} \ldots \varepsilon_{r}^{m_{r}} \vartheta_{1}^{n_{1}} \ldots \vartheta_{s}^{n_{s}} u_{1}^{k_{1}} \ldots u_{w}^{k_{w}} X^{q}
$$

where $X \in \mathcal{O}_{\mathbf{k}}, \max \left|m_{i}\right|<q$ and $\varepsilon_{0}, \ldots, \varepsilon_{r}$ are units such that $\max \left\{\left[\varepsilon_{0}, \ldots, \sqrt{\varepsilon_{r}}\right\}\right.$ is bounded.

By similar arguments we have

$$
\left(y_{1}+z^{p}\right)^{h}=\varepsilon_{0}^{i_{0}} \ldots \varepsilon_{r}^{i_{r}} \vartheta_{1}^{j_{1}} \ldots \vartheta_{s}^{j_{s}} v_{1}^{l_{1}} \ldots v_{t}^{l_{t}} Y^{p}
$$

where $Y \in \mathcal{O}_{k},\left|l_{i}\right| \leqslant n, \max \left\{\left|i_{0}\right|, \ldots,\left|i_{r}\right|,\left|j_{1}\right|, \ldots,\left|j_{s}\right|\right\}<p$ and $\max \left|v_{i}\right|<c_{20} q$.
Firstly we aim to show that

$$
q<\chi_{1}(\log p)^{\chi_{2}}
$$

for some effective constants $\chi_{1}$ and $\chi_{2}$. We suppose that

$$
A=\left(x_{1}-z^{q}\right)^{h p}\left(y_{1}+z^{p}\right)^{-h q}-1 \neq 0
$$

(later, we shall deal with case $A=0$ ). By construction of the element $z$ we have $\mathfrak{p}_{i}^{\alpha_{i}}\left|[z], \mathfrak{p}_{i}^{\alpha_{i} q}\right|[z]^{p}, z^{p q} \mid x_{1}^{h p}-y_{1}^{h q}$ and $\mathfrak{p}_{i} \chi\left[y_{1}+z^{p}\right]$. It means that (using the notation introduced between Lemma 2 and Lemma 3)

$$
|\Lambda|_{\mathfrak{p}_{i}} \leqslant\left(N \mathfrak{p}_{i}\right)^{-\alpha_{i} q / e_{p_{i}} \delta_{\mathfrak{p}_{i}}} \leqslant\left(N p_{i}\right)^{-\alpha_{i} q^{n}-1} .
$$

Now, we shall apply Lemma 2 to $\Lambda$ with $k=r+s+w+t+2, B=2 p^{2}$, $\alpha_{k}=X Y^{-1}$,

Since

$$
\left\{\alpha_{1}, \ldots, \alpha_{k-1}\right\}=\left\{\varepsilon_{0}, \ldots, \varepsilon_{r}, \vartheta_{1}, \ldots, \vartheta_{s}, u_{1}, \ldots, u_{w}, v_{1}, \ldots, v_{t}\right\} .
$$

and

$$
\begin{aligned}
& \max \left\{H\left(\varepsilon_{0}\right), \ldots, H\left(\varepsilon_{r}\right), H\left(\vartheta_{1}\right), \ldots, H\left(\vartheta_{s}\right)\right\}<c_{21}, \\
& \max \left\{H\left(u_{1}\right), \ldots, H\left(u_{w}\right), H\left(v_{1}\right), \ldots, H\left(v_{v}\right)\right\}<p^{c_{22}}
\end{aligned}
$$

$$
H\left(X Y^{-1}\right) \leqslant(|X|+\mid Y)^{n} \leqslant M^{2 n},
$$

where $M=\max \{|\mathbb{X}, \sqrt{Y}|, 2\}$, we have

$$
|\Lambda|_{p_{i}}>\exp \left\{-c_{23}(\log p)^{c 24} \log M\right\} \text {. }
$$

Comparing (8) with (9) we obtain

$$
\left(N p_{i}\right)^{\alpha_{i} q}<M^{c_{25}(\log p)^{c_{24}}} \quad\left(c_{24}>1\right) \text {. }
$$

As we want to show that $q<\chi_{1}(\log p)^{\chi_{2}}$ we may assume that $M>c_{26}$ where $c_{26}$ is to be determined later. Taking the product of the inequalities (10) for all $\mathfrak{p}_{i}(i=1, \ldots, s)$ we get

and

$$
N^{q}\left(p_{1}^{\alpha_{1}} \ldots: p_{s}^{\alpha_{s}}\right)<M^{c_{27}(\log p)^{c}}
$$

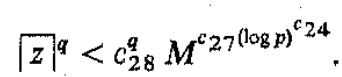

Supposing $q>12 c_{27} h(\log p)^{c_{24}}$ and $c_{26}>c_{28}^{12 h}$ we have

$$
\left\lceil z<M^{1 / 6 h}\right. \text {. }
$$

Write

and

$$
U=\varepsilon_{0}^{m_{0}} \ldots \varepsilon_{r}^{m_{r}} \vartheta_{1}^{n_{1}} \ldots \vartheta_{s}^{n_{s}} u_{1}^{k_{1}} \ldots u_{w}^{k_{w}}
$$

$$
V=\varepsilon_{0}^{i_{0}} \ldots \varepsilon_{r}^{i_{r}} \vartheta_{1}^{j_{1}} \ldots \vartheta_{s}^{j_{s}} v_{1}^{l_{1}} \ldots v_{t}^{l_{t}}
$$


Then $|U|<c_{29}^{q},|V|<c_{30}^{p}$ and

$$
U^{(j)}=|N(U)| \prod_{i \neq j}\left|U^{(i)}\right|^{-1}>c_{31}^{-q}, \quad j=1, \ldots, n,
$$

and similarly

$$
V^{(j)}>c_{32}^{-p}, \quad j=1, \ldots, n .
$$

Using (5), (6) and (12) we obtain at least one of the following inequalities

$$
\left|x_{1}>\right| X^{q / h} c_{31}^{-q}-|z|^{q}>M^{q / h} c_{31}^{-q}-M^{q / 6 h}
$$

hold.

$$
\left|y_{1}>\right| Y^{p / h} c_{32}^{-p}-|z|^{p}<M^{p / h} c_{32}^{-p}-M^{p / 6 h}
$$

Supposing $M>\max \left\{c_{31}^{2 h}, c_{32}^{2 h}\right\}$ and $q>6 h$ we get

$$
\left\lceil x_{1}\right\rceil>M^{q / 3 h}
$$

or

$$
\left\lceil y_{i}^{i}>M^{p / 3 h}\right. \text {. }
$$

If $\left\lceil x_{1}\right\rceil>M^{q / 3 h}\left(>\bar{z}^{2 q}\right)$ then

$$
\left|y_{1}^{(j)}\right|^{q} \geqslant\left|x_{1}^{(j)}\right|^{p}-\left|z^{(j)}\right|^{p q}, \quad j=1, \ldots, n
$$

and

$$
\left.\bar{y}_{1}\right|^{q} \geqslant\left|\bar{x}_{1}\right|^{p}-|z|^{p q}>M^{p q / 3 h}-M^{p q / 6 h}>M^{p q / 4 h}>\left.\right|^{3 p q / 2} .
$$

In the other case, when $\mid y_{1}>M^{p / 3 h}$, we have by a similar argument

$$
\left\lceil\left. x_{1}\right|^{p}>|\bar{z}|^{3 p q / 2}\right. \text {. }
$$

So, we may assume that

$$
\min \left\{\left[{\overline{x_{1}}}^{p},{\overline{y_{1}}}^{q}\right\}>M^{p q / 4 h}>|z|^{3 p q / 2} .\right.
$$

Choose $j$ such that $\left|x_{1}\right|=\left|x_{1}^{(j)}\right|$. Then we have

$$
\left|\left(\frac{x_{1}^{(j)}-\left(z^{(j)}\right)^{q}}{x_{1}^{(j)}}\right)^{h p}-1\right| \leqslant\left(\begin{array}{c}
h p \\
1
\end{array}\right) \frac{{ }^{\mid q^{q}}}{\sqrt{x_{1}}}+\ldots+\left(\begin{array}{c}
h p \\
h p
\end{array}\right) \frac{[]^{h p q}}{\sqrt{\left.x_{1}\right|^{p p}}} .
$$

Since we intend to show that $q<\chi_{1}(\log p)^{\chi_{2}}$ we may assume that $(h p)^{2}<M^{q / 12 h}$.

Then by (13)

$$
\sqrt{x_{1}} / / \bar{z}^{q}>M^{q / 4 h} / M^{q / 6 h}(h p)^{2},
$$

hence

$$
\left(\begin{array}{c}
h p \\
i
\end{array}\right) \frac{\sqrt{z^{a i}}}{\left|x_{1}\right|^{i}} \geqslant\left(\begin{array}{c}
h p \\
i+1
\end{array}\right) \frac{\sqrt{z^{(i+1)}}}{\left|x_{1}\right|^{i+1}}, \quad i=1, \ldots, h p-1
$$

Therefore from (11) we obtain

$$
\begin{aligned}
\left|\left(\frac{x_{1}^{(j)}-\left(z^{(j)}\right)^{q}}{x_{1}^{(j)}}\right)^{h p}-1\right| & <(h p)^{2} \frac{\sqrt{\left.z\right|^{q}}}{\sqrt{x_{1}}}<\frac{M^{q / 12 h} c_{28}^{q} M^{c_{2} 27^{(\log p)^{c}}}}{M^{q / 4 h}} \\
& <\frac{M^{c_{27} 7^{(\log p)^{c}}}}{M^{c_{33 q}}} .
\end{aligned}
$$

Now, we shall give an upper bound for

$$
\left|\left(y_{1}^{(j)}\right)^{h q}\left(y_{1}^{(j)}+\left(z^{(j)}\right)^{p}\right)^{-h q}-1\right| .
$$

By taking $c_{17}(<q)$ large enough we obtain

$$
\begin{aligned}
\left|\frac{y_{1}^{(j)}}{\mid\left(z^{(j)}\right)^{p}}+1\right| & >\left|\frac{y_{1}^{(j)}}{\mid\left(z^{(j)}\right)^{p}}\right|-1=\left|\frac{\left(x_{1}^{(j)}\right)^{p}}{\left(z^{(j)}\right)^{p q}}-1\right|^{1 / q}-1 \\
& >\left(\frac{\mid x_{1}}{\left.2 \sqrt{z}\right|^{q}}\right)^{p / q}-1>\frac{\mid x_{1}}{\left.4 \sqrt{z}\right|^{q}}>\frac{M^{q / 4 h}}{4 M^{q / 6 h}}>(h q)^{2} .
\end{aligned}
$$

Then

$$
\left(\begin{array}{c}
h q \\
i
\end{array}\right) \frac{\left(4 \mid{ }^{q}\right)^{i}}{\left|x_{1}\right|^{i}}>\left(\begin{array}{c}
h q \\
i+1
\end{array}\right) \frac{\left.(4 \sqrt{z})^{q}\right)^{i+1}}{\left|x_{1}\right|^{i+1}}, \quad i=1, \ldots, h q-1
$$

and therefore

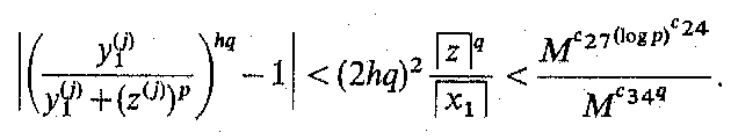

Since

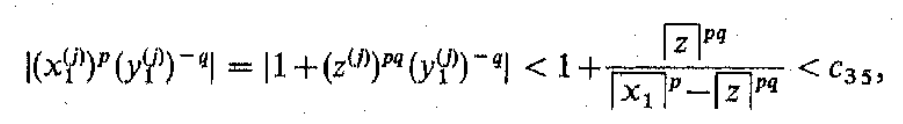

we have, by (11),

$$
\begin{aligned}
\left|\frac{\left(x_{1}^{(j)}\right)^{h p}}{\left(y_{1}^{(j)}\right)^{h q}}-1\right| & <c_{36} \frac{c_{28}^{p q} M^{c_{27} 7^{(\log p)^{c}}}}{M^{c_{3} 7_{p q} q}}<\frac{M^{c_{27} p(\log p)^{c_{24}}}}{M^{c_{38} 8^{p q}}} \\
& <\frac{M^{c_{27}(\log p)^{c_{24}}}}{M^{c^{38 q}}}
\end{aligned}
$$

if $M$ is large enough and $c_{27}(\log q)^{c_{24}}<c_{38} q$.

For any complex numbers $z_{1}, z_{2}, z_{3}$

$$
z_{1} z_{2} z_{3}-1=\prod_{i=1}^{3}\left(z_{i}-1\right)+\sum_{1 \leqslant i<j \leqslant 3}\left(z_{i}-1\right)\left(z_{j}-1\right)+\sum_{i=1}^{3}\left(z_{i}-1\right) .
$$


Using (14), (15) and (16) we obtain

$$
\left|\left(x_{1}^{(j)}-\left(z^{(j)}\right)^{q}\right)^{h p}\left(y_{1}^{(j)}+\left(z^{(j)}\right)^{p}\right)^{-h q}-1\right|<\frac{M^{c^{39} 9^{(\log p)^{c_{40}}}}}{M^{c_{41} q}}
$$

On the other hand, from Lemma 1 we have

$$
\left|\Lambda^{(j)}\right|>\exp \left(-c_{42}(\log p)^{c_{43}} \log M\right) .
$$

Comparing (17) with (18) we see that

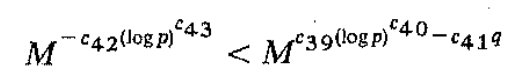

and this yields $q<\chi_{1}(\log p)^{\chi_{2}}$.

We assumed that $A \neq 0$; supposing the contrary we have

$$
\left(x_{1}-z^{q}\right)^{h p}=\left(y_{1}+z^{p}\right)^{h q} \text {. }
$$

If $\mathfrak{p} \mid\left[x_{1}-z^{q}\right]$ for some prime ideal $\mathfrak{p}$ then, by (2),

$$
p\left|\left[x_{1}^{p}-z^{p q}\right], \quad \mathfrak{p}\right|\left[y_{1}\right] \quad \text { and } \quad p \mid\left[y_{1}+z^{p}\right] .
$$

It means that $p \mid q_{1} \ldots q_{s}$. Put

$$
\mathfrak{q}_{1} \ldots \mathfrak{q}_{s}=\mathfrak{R}_{1}^{\delta_{1}} \ldots \mathfrak{R}_{s_{1}}^{\delta_{s_{1}}}
$$

where $\mathfrak{R}_{1}, \ldots, \mathfrak{R}_{s_{1}}$ are distinct prime ideals. Then

$$
\begin{aligned}
& {\left[x_{1}-z^{q}\right]=\left(\mathfrak{R}_{1}^{\tau_{1}} \ldots \mathfrak{R}_{s_{1}}^{\tau_{s_{1}}}\right)^{q},} \\
& {\left[y_{1}+z^{p}\right]=\left(\mathfrak{R}_{1}^{\omega_{1}} \ldots \mathfrak{R}_{s_{1}}^{\omega_{s_{1}}}\right)^{p} .}
\end{aligned}
$$

where $\tau_{1}, \ldots, \tau_{s_{1}}, \omega_{1}, \ldots, \omega_{s_{1}}$ are non-negative integers. We may assume that $(p q, h)=1$. Therefore $\mathfrak{R}_{1}^{\tau_{1}} \ldots \mathfrak{R}_{s_{1}}^{\tau_{s_{1}}}$ and $\mathfrak{R}_{1}^{\omega_{1}} \ldots \mathfrak{R}_{s_{1}}^{\omega_{s_{1}}}$ are principal ideals and we have as before (cf, (5))

$$
\begin{aligned}
& x_{1}-z^{q}=\varepsilon_{0}^{\mu_{1}} \ldots \varepsilon_{r}^{\mu_{r}} X_{1}^{q}, \\
& y_{1}+z^{p}=\varepsilon_{0}^{v_{1}} \ldots \varepsilon_{r}^{v_{r}} Y_{1}^{p}
\end{aligned}
$$

for some $X_{1}, Y_{1} \in \mathscr{O}_{K}$ and $\max _{i}\left|\mu_{i}\right|<q, \max _{i}\left|v_{i}\right|<p$. Now, we show that

$$
A_{1}=\left(x_{1}-z^{q}\right)^{p}\left(y_{1}+z^{p}\right)^{-q}-1 \neq 0 \text {. }
$$

Indeed, if

$$
\left(x_{1}-z^{q}\right)^{p}=\left(y_{1}+z^{p}\right)^{q}
$$

then

(19)

$$
\left(\begin{array}{l}
p \\
1
\end{array}\right) x_{1}^{p-1} z^{q}=x_{1}^{p}-y_{1}^{q}+\beta z^{q+1}+\gamma z^{p}
$$

for some $\beta, \gamma \in \mathcal{O}_{\boldsymbol{K}}$. From (19) we get

$$
z \mid x_{1}^{p-1} p
$$

By taking $c_{17}(<p)$ large enough we have

$$
p_{1} \times[p], \quad p_{1} \chi\left[x_{1}\right] \text { and } p_{1}^{\alpha_{1} q_{1}} \|\left[p x_{1}^{p^{-1}}\right]
$$

which contradicts $(20)$.

Now, we can repeat the arguments with $\Lambda_{1}$ instead of $\Lambda$, starting from $\left(5^{\prime}\right)$ and $\left(6^{\prime}\right)$ in place of (5) and (6), respectively, and we again arrive at (7), but with other constants.

In second part of the proof we shall show that

$$
p<\chi_{3}(\log p)^{\chi_{4}}
$$

where $\chi_{3}$ and $\chi_{4}$ are effectively computable constants. Write

(22)

$$
\Lambda_{2}=x_{1}^{p h}\left(y_{1}+z^{p}\right)^{-q h}-1 .
$$

We postpone discussion of the case $\Lambda_{2}=0$. Since $p_{i}^{\alpha_{i}} \mid[z]$ and $z^{p} \mid x_{1}^{p h}-\left(y_{1}+z^{p}\right)^{q h}$ but $p_{i} \chi\left[y_{1}+z^{p}\right]$, we obtain

$$
\left|\Lambda_{2}\right|_{p_{i}} \leqslant\left(N p_{i}\right)^{-\alpha_{i} p / e_{p_{i}} f_{p_{i}}} \leqslant\left(N p_{i}\right)^{-\alpha_{i} p / n}
$$

On the other hand $A_{2}$ can be written in the form (cf. (6))

$$
\Lambda_{2}=\varepsilon_{0}^{-i_{0} q} \ldots \varepsilon_{r}^{-i_{r} q} \vartheta_{1}^{-j_{1} q} \ldots \vartheta_{s}^{-j_{s} q} v_{1}^{-l_{1} q} \ldots v_{t}^{-l_{r} q}\left(x_{1}^{h} Y^{-q}\right)^{p}-1
$$

and we may apply Lemma 2 to give a lower bound for $\left|\Lambda_{2}\right|_{p_{\mathfrak{i}}}$. Putting

$$
M_{1}=\max \{[|X|,|Y|,|z|\}
$$

we have by (5)

hence

$$
\left|x_{1}\right|<c_{44}^{q} M_{1}^{q} p^{c_{45}},
$$

$$
H\left(x_{1}^{h} Y^{-q}\right) \leqslant\left({\overline{x_{1}}}^{h}+\left|{ }_{Y}\right|^{q}\right)^{n}<c_{46}^{q} M_{1}^{c_{47}^{q}} p^{c_{48}} .
$$

Therefore, by (7),

$$
\log H\left(x_{1}^{h} Y^{-q}\right)<c_{49}(\log p)^{c 50} \log M_{1} .
$$

Consequently, by Lemma 2 we obtain

$$
\left|\Lambda_{2}\right|_{p i}>\exp \left(-c_{51}(\log p)^{0_{52}} \log M_{1}\right)
$$

Comparing (23) with (25) we have

$$
2^{p_{i}} \leqslant\left(N p_{i}\right)^{\alpha_{i} p}<M_{1}^{c 3^{(\log p)^{c}}} \cdot
$$

As we aim to show (21) we may assume that $M_{1}>c_{53}$ where $c_{53}$ is to be 
determined later. Taking the product of inequalities (26) we have

$$
\left.\left.\right|^{p}\right\rceil^{p} \leqslant\left(c_{3} N(z)\right)^{p}<c_{54}^{p} M_{1}^{c_{55}(\log p)^{c_{5}}} \text {. }
$$

By assuming $p /(12 h)>c_{55}(\log p)^{c_{52}}$ and $c_{54}^{12 h}<c_{53}$ we obtain

$$
|z|<c_{54} M_{1}^{1 / 12 h}<M_{1}^{1 / 6 h}
$$

and by definition of $M_{1}$ we have

and

$$
M_{1}=M \quad(=\max \{|X|,|Y|\})
$$

$$
|z|<M^{1 / 6 h} \quad \text { (cf. (12)) }
$$

Hence, we may assume that

$$
\min \left\{x_{x_{1}}^{p},{ }_{y_{1}}^{q}\right\}>M^{p q / 4 h}>\left.\right|_{z} ^{3 p q / 2} \quad \text { (cf. (13)). }
$$

By $\left|x_{1}^{(j)}\right|=\mid x_{1}$ we get

Consequently,

$$
\left|x_{1}^{(j)}\right|^{p} \leqslant\left|y_{1}^{(j)}\right|^{q}+\left.\sqrt{z}\right|^{p q}, \quad\left[\left.\overline{y_{1}}\right|^{q} \leqslant\left.\dot{\mid x_{1}}\right|^{p}+\left.\sqrt{z}\right|^{p q} .\right.
$$

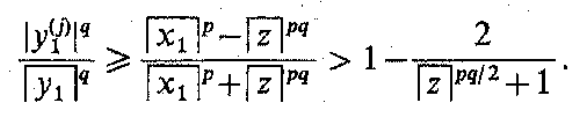

By Lemma 9 we have $|z|>1+c_{56}$ ( $z$ is not a root of unity because of $\left.p_{1} \mid[z]\right)$. Therefore by taking $c_{17}(<p)$ large enough we obtain $\left|y_{1}^{(f)}\right|>\frac{1}{2} \mid y_{1}$ and

$$
\begin{aligned}
& \left|\left(y_{1}^{(j)}\right)^{h q}\left(y_{1}^{(j)}+\left(z^{(j)}\right)^{p}\right)^{-h q}-1\right| \leqslant \sum_{i=1}^{h q}\left(\begin{array}{c}
h q \\
i
\end{array}\right) \frac{\sqrt{\left.z\right|^{p i}}}{\mid y_{1}^{(j)}+\left(z^{(j)}\right)^{\left.p\right|^{i}}} \\
& \sum_{i=1}^{h q}\left(\begin{array}{c}
h q \\
i
\end{array}\right) \frac{\sqrt{\left.z\right|^{p i}}}{\left.\left(\frac{1}{2} \mid y_{1}-\sqrt{z}\right)^{p}\right)^{i}}<\sum_{i=1}^{h q}\left(\begin{array}{c}
h q \\
i
\end{array}\right) \frac{\bar{z}^{p i}}{\left(\frac { 1 } { 2 } \left[y_{1}-\sqrt{\left.\left.y_{1}\right|^{2 / 3}\right)^{i}}\right.\right.} \\
& <\sum_{i=1}^{h q}\left(\begin{array}{c}
h q \\
i
\end{array}\right)\left(\left.{ }_{z}\right|^{p}{\overline{y_{1}}}^{-2 / 3}\right)^{i}<2^{h q} \frac{{ }_{z}^{p}}{\left|y_{1}\right|^{2 / 3}} \\
& <\frac{2^{k q} c_{54}^{p} M^{c_{55}(\log p)^{c 52}}}{M^{p / 6 h}} \\
& <\frac{M^{c 55^{(\log p)^{c 52}}}}{M^{c_{57 p}}} .
\end{aligned}
$$

Further, since $\left|\left(x_{1}^{(j)}\right)^{p}\left(y_{1}^{(j)}\right)^{-q}\right|<2$, we have

(28) $\left|\left(x_{1}^{(j)}\right)^{p h}\left(y_{1}^{(j)}\right)^{-q h}-1\right|<c_{58}\left|\left(x_{1}^{(j)}\right)^{p}-\left(y_{1}^{(j)}\right)^{q}\right|\left|y_{1}^{(j)}\right|^{-q}<\left.2^{q} c_{58}\right|^{z^{p q}} \mid y_{1}{ }^{-q}$

$$
\begin{aligned}
& <c_{58} 2^{q} c_{54}^{p q} M^{c_{55} q(\log p)^{c 52}} M^{-p q / 4 h} \\
& <M^{c_{59}(\log p)^{c} 60^{-c_{61} p}}
\end{aligned}
$$

For any complex numbers $z_{1}, z_{2}$

$$
z_{1} z_{2}-1=\left(z_{1}-1\right)\left(z_{2}-1\right)+\left(z_{1}-1\right)+\left(z_{2}-1\right)
$$

From (27) and (28) we have

$$
\left|\Lambda_{2}^{(j)}\right|<M^{c} 2^{(\log p)^{c} 63-c_{64} p}
$$

Moreover, by Lemma 1, we obtain

$$
\left|\Lambda_{2}^{(j)}\right|>\exp \left(-c_{65}(\log p)^{c_{66}} \log M\right) \text {. }
$$

Comparing (29) with (30) we have $p<\chi_{3}(\log p)^{\chi_{4}}$ or $q<p<c_{67}$. Finally, by virtue of Lemma $4, \max \{H(x), H(y)\}<c_{68}$.

To complete our argument we consider the case $\Lambda_{2}=0$. Then

$$
x_{1}^{p h}=\left(y_{1}+z^{p}\right)^{h q}
$$

and

$$
-\left(\begin{array}{c}
h q \\
1
\end{array}\right) y_{1}^{h q-1} z^{p}=y_{1}^{h q}-x_{1}^{h p}+E z^{2 p}=F z^{2 p}
$$

for some $E, F \in \mathscr{O}_{\mathfrak{K}}$. Since $\mathfrak{p}_{1} \mid[z]$ and $\mathfrak{p}_{1} \chi\left[y_{1}\right]$; we get $p_{1}^{p} \mid[h q]$ and

$$
2^{p} \leqslant\left(N p_{1}\right)^{p} \leqslant|N(h q)|<c_{69} p^{c_{70}}
$$

which implies $p<c_{71}$.

Added in proof. Recent work of Yu Kunrui (Linear forms in logarithms in the p-adic case, to appear in the Proceedings of the Durham conference on Transcendental Number Theory, 1986) has thrown doubt on the validity of van der Poorten's proof of Lemma 2. The matters in question, however, could only affect the constant $C_{p}$ and thus do not affect the results of this paper. In particular, observe that in the present work we may assume that we have the strong independence condition required according to $\mathrm{Yu}$, in order that the inequalities of $\mathrm{J}$. $\mathrm{H}$. Loxton and A. J van der Poorten (Multiplicative independence in number fields, Acta Arith. 42 (1983) pp. 291-302) allow us to appropriately transform the expressions to which we apply Lemma 2.

\section{References}

[1] A. Baker, The theory of linear forms in logarithms, in: Transcendence Theory: Advances and applications, Academic Press, London and New York 1977, pp. 1-27.

[2] B. Brindza, On S-integral solutions of the equation $f(x)=y^{m}$, Acta Math. Acad. Sci. Hung. 44 (1-2) (85), pp. 133-139.

[3] B. Brindza, K. Györy and R. Tijdeman, On the Catalan equation over algebraic number fields (to appear).

[4] D. G. Cantor and E. G. Straus, On a conjecture of. D. H. Lehmer, Acta Arith. 42 (1982), pp. 97-100. Correction, ibid. p. 327.

[5] J. W. S, Cassels, On the equation $a^{x}-b^{y}=1$, Amer. J. Math. 75 (1953), pp. 159-162

[6] E. Catalan, Note extraite d'une lettre adressée à Péditeur, J. Reine Angew, Math. 27 (1844), p. 192 
7] E. Dobrowolski, On a question of Lehmer and the number of irreducible factors of a polynomial, Acta Arith. 34 (1979), pp. 391-401.

[8] K. Györy, On the solutions of linear diophantine equations in algebraic integers of bounded norm, Ann. Univ. Sci. Budapest, Eötvös, Sect. Math. 22-23 (1979-1980), pp. 225-233.

[9] - Résultats effectifs sur la représentation des entiers par des formes décomposables, Queen's Papers in Pure and Applied Mathematics, No 56, Kingston, Canada, 1980.

[10] - On the number of solutions of linear equations in units of an algebraic number field, Comment. Math. Helvetici 54 (1979), pp. 583-600.

[11] W. J. LeVeque, On the equation $y^{m}=f(x)$, Acta Arith, 9 (1964), pp. 209-219.

[12] A. J. van der Poorten, Effectively computable bounds for the solutions of certain diophantine equations, ibid. 33 (1977), pp. 197-206.

[13] P. Ribenboim, Consecutive powers, Exposit. Math, 2 (1984), pp. 193-221

[14] T. N. Shorey and R. Tijdeman, Exponential diophantine equations, Cambridge University Press, New York etc., 1987.

[15] R. Tijdeman, On the equation of Catalan, Acta Arith. 29 (1976), pp. 197-209

[16] - On the Fermat-Catalan equation, Jahresber. Deutsche Math. Verein. 87 (1985) pp. 1-18.

MATHEMATICAL INSTITUTE

KOSSUTH LAJOS UNIVERSTT

4010 Debrecen Pf. 12. Hungary

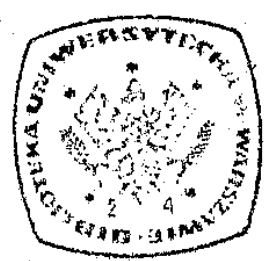

